\begin{tabular}{|c|c|}
\hline Title & Quantitative visualization of vortex ring structure during wall impingement subject to background rotation \\
\hline Author(s) & Oishi, Y oshihiko; Murai, Y uichi; Tasaka, Y uji \\
\hline Citation & $\begin{array}{l}\text { Journal of visualization, 22(5), 867-876 } \\
\text { https://doi.org/10.1007/s12650-019-00575-4 }\end{array}$ \\
\hline Issue Date & $2019-10$ \\
\hline DOC URL & http:/hdl.handle.net/2115/79358 \\
\hline Rights & $\begin{array}{l}\text { This is a post-peer-review, pre copyedit version of an article published in Journal of visual ization. The final } \\
\text { authenticated version is available online at: http://dx.doi.org/10.1007/s12650-019-00575-4. }\end{array}$ \\
\hline Type & article (author version) \\
\hline File Information & JOVI2019-Oishi-ver_final.pdf \\
\hline
\end{tabular}

Instructions for use 
Yoshihiko Oishi ${ }^{1}$, Yuichi Murai ${ }^{2}$, Yuji Tasaka²

\title{
Quantitative visualization of vortex ring structure during wall-impingement subject to background rotation
}

\author{
${ }^{1}$ Division of Production Systems Engineering, Muroran Institute of Technology, Muroran, Japan \\ ${ }^{2}$ Laboratory for Flow Control, Faculty of Engineering, Hokkaido University, Sapporo, Japan \\ Corresponding Author: Yoshihiko Oishi, Assistant Professor, Dr. \\ E-mail: oishi@rmmm.muroran-it.ac.jp, Ph: +81(Japan)-143-46-5374
}

\begin{abstract}
A single vortex ring subject to background rotation in the process of wall-impingement has been experimentally investigated by particle tracking velocimetry (PTV). Two parameter conditions of Reynolds and Rossby number were chosen in addition to stationary environment as much strong and competitive Coriolis force emerges in comparison with inertia induced by vortex rings. From horizontal PTV windows set on the rotating experimental frame above the bottom wall, comprehensive influences of Coriolis force on the wall-impinging reaction are visualized as space-time three-dimensional vorticity distributions. Against natural growth of azimuthal waves due to Widnall instability, wall-impinging suppresses the waves and rather re-organizes original primary vortex because of cyclonic swirl coherently induced during impingement. This resists to turbulent collapse of vortex ring during the impingement, and self-boosts own life time. We try to explain the mechanism of such an anti-decaying process in the final part, untangling the phenomenon with best read from the space-time correlations among three vorticity components.
\end{abstract}

Keywords Vortex ring, rotating flow, impingement, PTV and 3D vortex structure 


\section{Introduction}

A vortex ring has a beautiful structure in fluid motions that has attracted attention of many researchers since the earliest work by Helmholtz in $19^{\text {th }}$ century. Many researches have been reported on its nature (e.g., Tung et al. 1967; Saffman 1970; Shariff et al. 1992; Weigand et al. 1994; Dziedzic and Leutheusser 1996; Gharib et al. 1998; Schram et al. 2001; Linden et al. 2001; Krueger et al. 2003; Krueger 2005; and Dazin et al. 2006). Beside viscous decay, sustainability of vortex rings is lost in non-quiescent environments, for example in the presence of shear and in rotation. Among the variety of environments, rotating backgrounds are regarded as the simplest environment to be studied. This is because background rotation is free from both shear strains and unsteadiness. The influence of rotation also explains how vortex rings survive within turbo-machineries as well as rotating planets. In the same meaning, wall impingement of a vortex ring has great importance in mechanical engineering, and provides rich physics on behaviors of vortex rings. We expect the present visualizations in situations combining background rotation and wall-impingement offer new insights of multi-polar vortex dynamics, i.e. topological flow transition of vortical structures in three-dimensional (3D) space.

As Auerbach (1988) reported, spatial development of a vortex ring in stationary environment is classified by four stages: initial ring formation from an annular shear layer (e.g. Glezer 1988;, Mohseni et al. 1998), stable laminar travelling process (e.g. Norbury 1973), azimuthal wave generation due to Widnall instability (Widnall et al. 1974), and turbulent dissipation before collapse. Influence of the background rotation provides different additional events within individual stages. Moffatt (1988) and Turkington (1989) theoretically investigated the influence on laminar travelling process, and predicted slow-down of the translational velocity upon vortex filament theorem. Verzicco et al. (1996) proved the slow-down effect experimentally and found secondary flows which further enhance the viscous dissipation. To see the influence on the formation process, Brend and Thomas et al. (2009) and Murai et al. (2013) constructed a large rotating water tank and observed a helical flow soon induced behind vortex ring, which destroys the toroidal structure to shorten the free-travelling distance. Using the same facility, Kitaura et al. (2010) measured the influence of background rotation and detected upstream secondary vortex that reduces the translational velocity. An opposite effect was found by Naitoh et al. (2014) and Gargan-Shingles et al. (2015) as they emitted fluid by a rotating cylinder into a stationary fluid. Such an initially swirling vortex shows a dynamical response different from non-swirling vortex ring in a rotating frame. We, therefore, reached at an understanding from above literatures that background rotation always destabilizes the primary structure of vortex rings in all the four stages from the birth until the collapse.

In wall-impinging process, however, we have found contrastive reaction of vortex rings owing to the rotation. Experiments of the wall-impingement reaction deepens our knowledge on the transport processes of mass, momentum, and thermal energy by vortex rings. In stationary fluid environment, vortex ring impinging 
a wall provides strong shear stress in the wall proximity, and immediately transits to turbulence. A number of perspective case studies have been reported to date such as Boldes et al. (1973), Walker et al. (1987), Orlandi et al. (1993), Chu et al. (1995), Luton et al. (1997), Swearingen et al. (1999), Naguib et al. (2004), and Xu et al. (2017). Yamada et al. (1982) and Walker et al. (1987) experimentally observed fingering of vortex ring after the impingement. Interaction with other type of solid objects was reported by Naaktgeboren et al. (2012), Cheng et al. (2014), and Mujal-Colilles et al. (2015) for permeable plates. Suzuki and Kumagai et al. (2007), Masuda et al. (2012), Bethke et al. (2012), and Misaka (2012) studied particulate layers transported by a vortex ring. For a heated wall, Arevalo et al. (2010)) found heat transfer promoted by the fingering patterns formed on the wall. Common discussion in these reports are rapid three-dimensionalization of vortex ring triggered by Widnall instability which produces azimuthal waves. In fact, such a rapid transition to turbulence is expected in engineering applications to painting (mass transfer), spot cooling (heat transfer), and local cleaning (momentum transfer) by shooting vortex rings toward a solid wall. Much of these effects are summarized by Martin (1977) and Jambunathan et al. (1992).

Question through the present study is how these effects are modified in a rotating frame. This paper reports that the wall-impinging process has a different path of the flow transition due to background rotation. From horizontal PTV window set on the experimental planar near wall, we found that rapid generation of cyclonic swirl inside the vortex ring rewrites the timeline of multi-polar vortex-vortex interaction which results in resisting to turbulent collapse.

\section{Experimental Methods}

We constructed a fully transparent cylindrical container put on a turntable. This enables us to observe vortex ring reactions optically in any direction. All the measurement instrumentations are mounted on the same rotating frame so that the flow behavior is visualized with influence of the background rotation. Technical details are elaborated below.

\subsection{Experimental Facility}

Our experimental facility for visualizing vortex ring impinging on a wall (Fig. 1) consists of three parts: a vortex ring generator, a double container, and a turntable. A piston-cylinder mechanism is employed as a vortex ring generator. The generator has $D_{P}=10 \mathrm{~mm}$ in diameter, and is mounted at the top of the facility submerging in water with $50 \mathrm{~mm}$. Vortex rings are emitted downward at the center of the container. The generator induces trapezoidal velocity profiles at the outlet so that a cylindrical shear layer is formed downstream. Piston stroke, $S_{P}$, is variable from 10 to $50 \mathrm{~mm}$, controlled by a PC. We fix the stroke at $S_{P}=25$ $\mathrm{mm}$ in this study, thus $S_{P} / D_{P}=2.5$, at which the vortex ring is most stably emitted. The distance from the outlet of the vortex ring generator to bottom wall of the tank is $100 \mathrm{~mm}$. Noted that each individual vortex ring has a slightly different behavior as they reach the bottom wall because of non-linearity arising from the 
Widnall instability. Furthermore, vortex rings often become inclined in their own migration displacing them from the central axis, especially near the collapse stage. Therefore, the propagation distance toward the wall needs to be a moderate length to keep the vortex rings in a visualization section near the bottom wall.

The double container has an inner cylindrical vessel and an outer rectangular tank. Both are fully transparent made of acrylic resins and open to atmosphere at the top. Diameter of the inner vessel is $190 \mathrm{~mm}$, and side length of the outer rectangular tank is $200 \mathrm{~mm}$. Water is filled in both containers as the water in the outer rectangular tank reduces optical distortion for imaging flows inside the inner vessel.

To rotate the whole facility, a powerful turntable consisted of an electric motor, gears, a controller, and double tables, was used. The double tables are formed by a lower table fixed with the main rotational shaft, and a higher table with a wide open-hole at the center. The double tables rotate in positive angular direction, i.e. counter-clockwise direction as being viewed from the top, the same as the rotation direction of the Earth on northern hemisphere. The rotational speed can vary from 0 to $2 \pi \mathrm{rad} / \mathrm{s}$, i.e. up to $1 \mathrm{~Hz}$ or $60 \mathrm{rpm}$. The experimental conditions are summarized in Table 1.

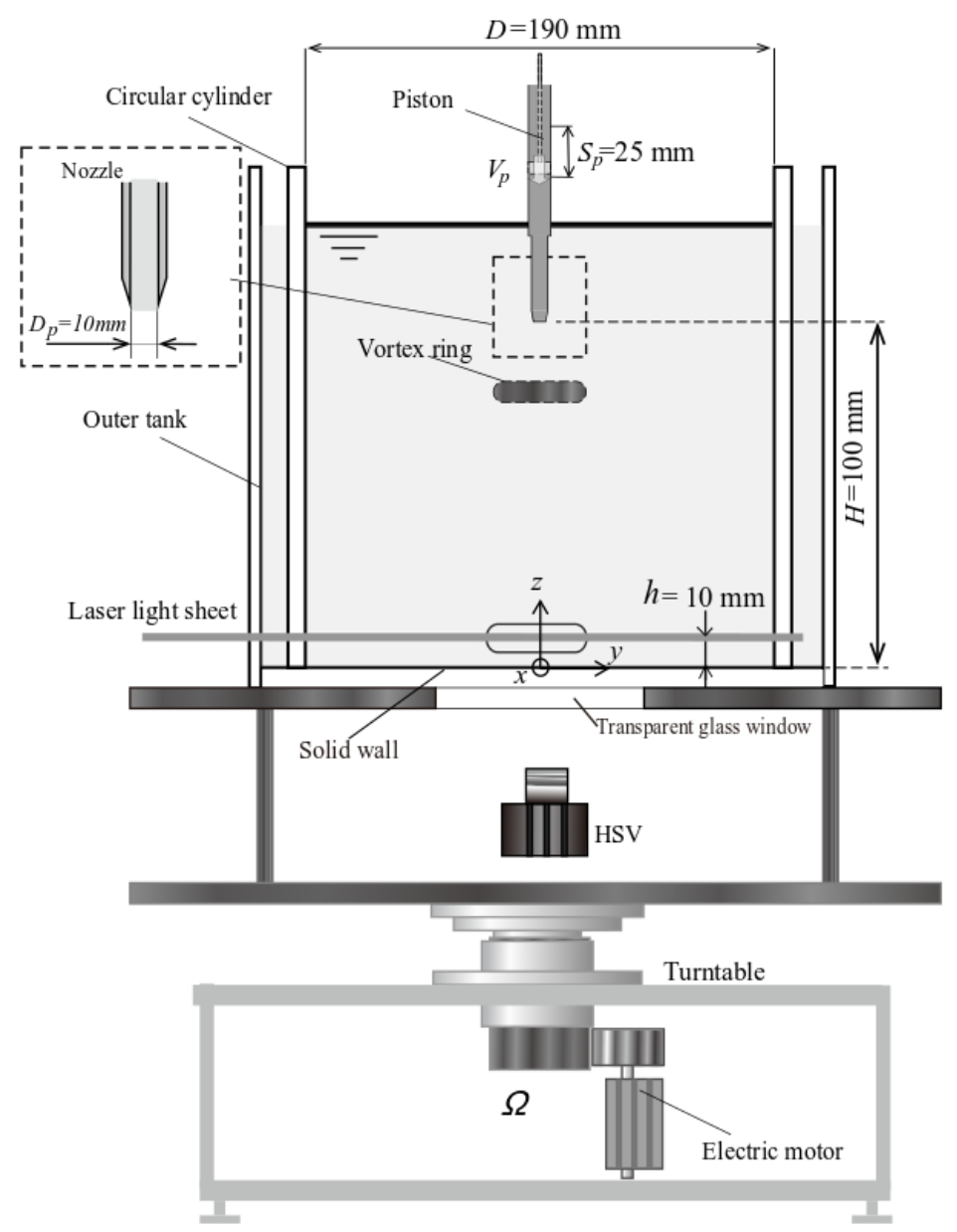

Fig. 1 Prospective view of experimental set-up for visualizing vortex ring structure interacting with a flat wall in a rotating container and visualization of wall-perpendicular structure. 
Table 1 Experimental conditions

\begin{tabular}{llll}
\hline Piston velocity & $V_{\mathrm{p}}$ & 300,600 & {$[\mathrm{~mm} / \mathrm{s}]$} \\
Kinematic viscosity & $N$ & 1.004 & {$\left[\mathrm{~mm}^{2} / \mathrm{s}\right]$} \\
Temperature & $T$ & 20 & {$\left[{ }^{\circ} \mathrm{C}\right]$} \\
Rotating speed of field & $\Omega$ & $0,0.52,1.05$ & {$[\mathrm{rad} / \mathrm{s}]$} \\
Reynolds number based on piston & $R e_{p}$ & 207,844 & \\
Reynolds number based on fluid & $R e_{F}$ & 103,422 & \\
Rossby number based on piston & $R o_{p}$ & 1.28 to infinity & \\
Rossby number based on fluid & $R o_{F}$ & 0.64 to infinity & \\
\hline
\end{tabular}

\subsection{Flow Visualization}

Flow visualization using tracer particles and PTV analysis on the particle images to quantify flow fields were adopted. In the set-up for horizontal illumination, the azimuthal structures close to the wall on $x-y$ plane are captured by a laser light sheet, $0.2 \mathrm{~W}$ YAG laser $(532 \mathrm{~nm})$ via a cylindrical lens, provided horizontally at the distance $h=10 \mathrm{~mm}$ from the bottom plane (Fig. 1). Thickness of the laser sheet is about $2 \mathrm{~mm}$ in Gaussian effective value, which was judged optimum as the flow has significant out-of-plane velocity. The technique applied is the same as for Taylor-Couette flow (Murai et al., 2018). As water flow tracer for the visualizations, high porous-type particles (DIAION, HP20. Mitsubishi Chemical Co. Ltd, Japan), which have mean diameter of $200 \mu \mathrm{m}$ and specific weight of 1.01, were employed. A high-speed digital video camera (EX-F1, Casio, Japan) is used to record the particle images. Image size is $1024 \times 1024$ pixels where the spatial resolution is $0.117 \mathrm{~mm} /$ pixel. Velocity resolution of PTV is estimated to be $0.035 \mathrm{~m} / \mathrm{s}$, which corresponds to $7 \%$ in maximum random error relative to the average traveling velocity of vortex rings. As our preliminary experiment, we confirmed that the primary vortex core did not penetrate the laser sheet on any condition due to natural bouncing at the wall.

Vortex ring has high spinning rate inside the primary vortical core, and high shear rate during wall impingement. Furthermore, multi-frame Lagrangian tracking of individual particles over three frames was quite difficult because of large out-of-plane velocity. According to the reasons above, for PTV algorithm, we employ a two-frame particle correlation technique termed velocity gradient tensor PTV (Ishikawa et al. (2000)), which has a robustness for strong fluid deformation. A higher order interpolation algorithm to provide velocity vectors arranged on regular grids from irregularly scattered velocity vector distributions as results of the PTV. The algorithm termed BER (biquadratic ellipsoidal rearrangement) produces a $\mathrm{C}^{3}$ continuous function of velocity vectors, which thus has spatial continuity of the first-order derivative such as vorticity (Ido et al. $(2002,2006)$ ). This is key point to have 3D isosurfaces of vorticity for understanding vortex structures detailed below. 

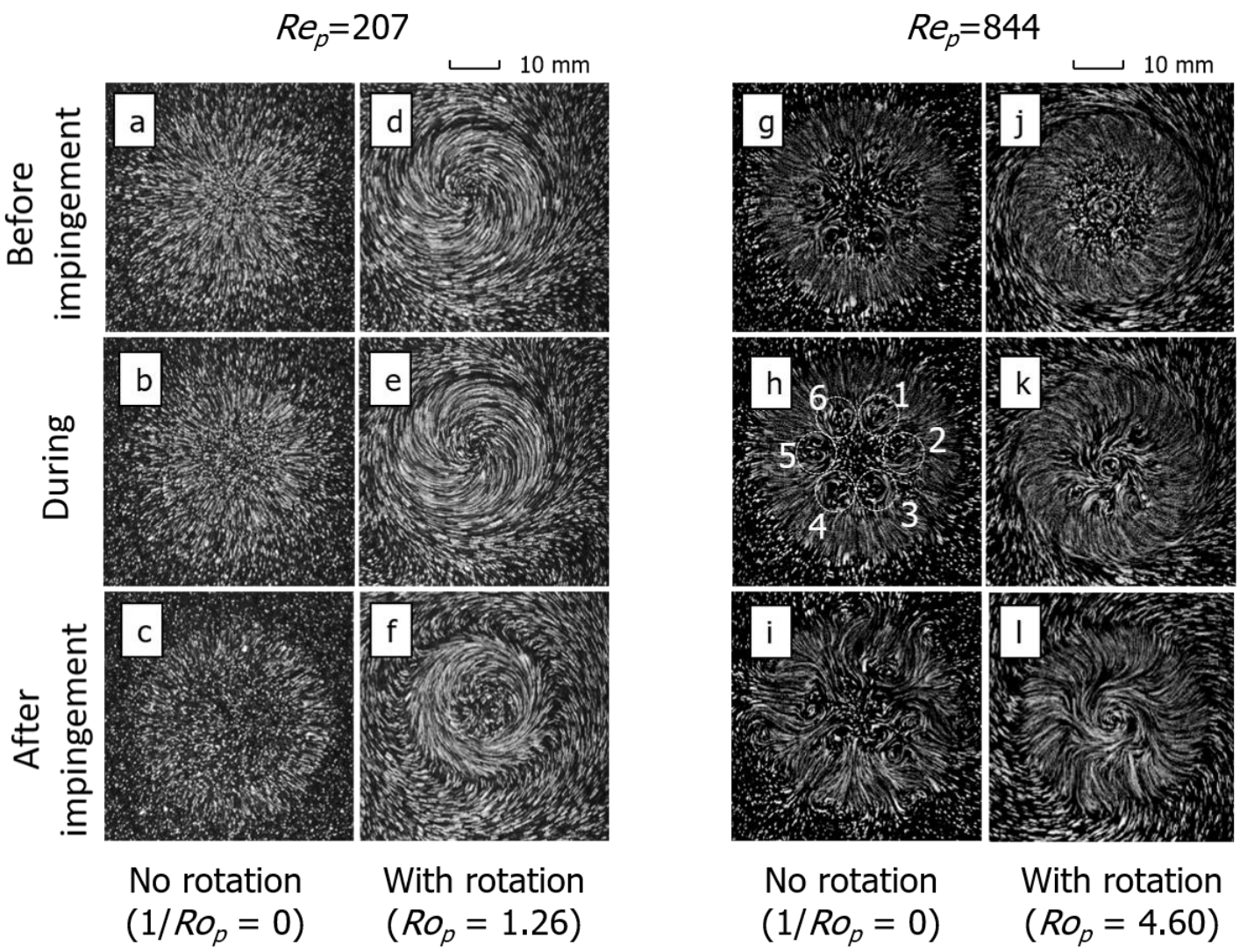

Fig. 2 Mono- and multi-swirl structures induced inside a vortex ring due to wall-impingement with background rotation, photographed as particle path-line image taken from the bottom window for visualizing flow in the $x-y$ plane. $R e_{P}$ and $R o_{P}$ numbers are defined by initial values at the piston.

\section{Experimental Results}

3.1 Visualization of azimuthal instability and parameter setting

Before presenting the results, here we define four dimensionless parameters describing vortex ring structures in a rotating system as below.

$$
R e_{P}=\frac{V_{p} D_{p}}{v}, \quad R e_{F}=\frac{V_{F} D_{F}}{v}, \quad R o_{P}=\frac{2 V_{p}}{\Omega D_{P}}, \quad R o_{F}=\frac{2 V_{F}}{\Omega D_{F}} .
$$

$R e$ and Ro mean Reynolds and Rossby numbers, respectively. The suffix $P$ denotes piston, and $F$ denotes fluid. Ordinarily, vortex rings generated by a piston-cylinder mechanism have a translational velocity of approximately $V_{F}=V_{P} / 2$, and $D_{F}=D_{P}$. We focus on the condition that vortex rings have competitive regime between the translational inertia and the centrifugal inertia, i.e. Rossby number ranges at $0.2<R o_{P}<\infty$. For sufficiently high background rotation $\left(R o_{P}<0.2\right)$, vortex rings are essentially unstable and do not reach the wall. Targeted range of the Reynolds number is $100<R e_{P}<1000$ at which toroidal ring structure appears with statistic axisymmetry. For $\operatorname{Re}_{P}$ numbers higher than the range, we found vortex rings being destabilized due to turbulent dissipation before impingement the wall. As background rotation was given for $\operatorname{Re}_{P}>3000$, position of the impingement on the bottom plate was highly fluctuated. With these facts, we eliminated such 
cases in data analysis. Besides, Widnall instability is inevitable during long free-travelling process. The instability usually induces a countable number of waves along the toroidal vortex core. In the process of wall-impingement, these waves play a significant role in reaction of vortex ring (Walker et al. 1987).

\subsection{Development of flow structures in impingement}

Internal structure of vortex rings is visualized by particle pathline imaging technique. Motion of the tracer particles at frame rate of $60 \mathrm{fps}$ in the wall-impingement moment of vortex rings was recorded. By adjusting the time integral period of frames, 20 frames in the following results, particle pathlines was obtained at the most visible average length. The pathline images recorded at moments, before, during and after the impingement, on the horizontal visualization plane are summarized in Fig. 2. Conditions of two Re numbers, $R e_{P}=207$ and 844 are focused on, where the latter shows clear azimuthal waves in impingement as shown in Fig. 2(h), while the former does not (Fig. 2(b)). Also two different $R o$ conditions are examined, $R o_{P}=1.26$ for $R e_{P}=207$ and $R o_{P}=4.60$ for $\operatorname{Re}_{P}=844$ in addition to stationary environment $\left(1 / R o_{P}=0\right)$, where the conditions provide relatively stronger and weaker Coriolis force relative to the inertia induced by the vortex ring.

For the low $R e$ number vortex ring (at $R e_{P}=207$ ), flow expanding in the radial direction is replaced with multiple layers of swirl structure as the background rotation is applied (see the panels (a) to (f) in Fig. 2). This is because that fluid moves inward in the section, and conservation of the angular momentum provides a cyclonic flow faster than the background rotation. The cyclonic swirl is maintained after the wall-impingement in the central part. Carefully looking at the pathline (b) on the non-rotating condition, a periodic deformation in the azimuthal direction is observable, which comes from Widnall instability. It infers that such a small structure is overshadowed and swept away by the cyclonic swirl as background rotation is given. At the high Re number (see the panels of (g) to (l)), contrastively, the azimuthal structure is more clearly observable than the low Re number cases as labeled in the panel (h), and it remains even with background rotation as identifiable in the panel (k). The region of cyclonic swirl is narrowed into the central axis, but it is rather intensified. Hence, background rotation produces a thin-localized, but high-spinning swirl at the central axis. Reproducibility of these phenomena was confirmed by repeating the visualization for several vortex rings on the same conditions.

For quantitative assessment of the vortical structure, PTV is applied. Figure 3 depicts two cases of PTV data on the $x-y$ plane at $R e_{P}=844$ with and without background rotation, $R o_{P}=4.60$ and $1 / R o_{P}=0$. Most of the fluid motion is concentrated inside the primary ring-shaped vortex core while the ambient fluid keeps stationary. Due to wall impingement, magnitude of velocity decreases in time. With background rotation, however, the magnitude is maintained after the impingement because of swirl motion induced in $x-y$ plane. 


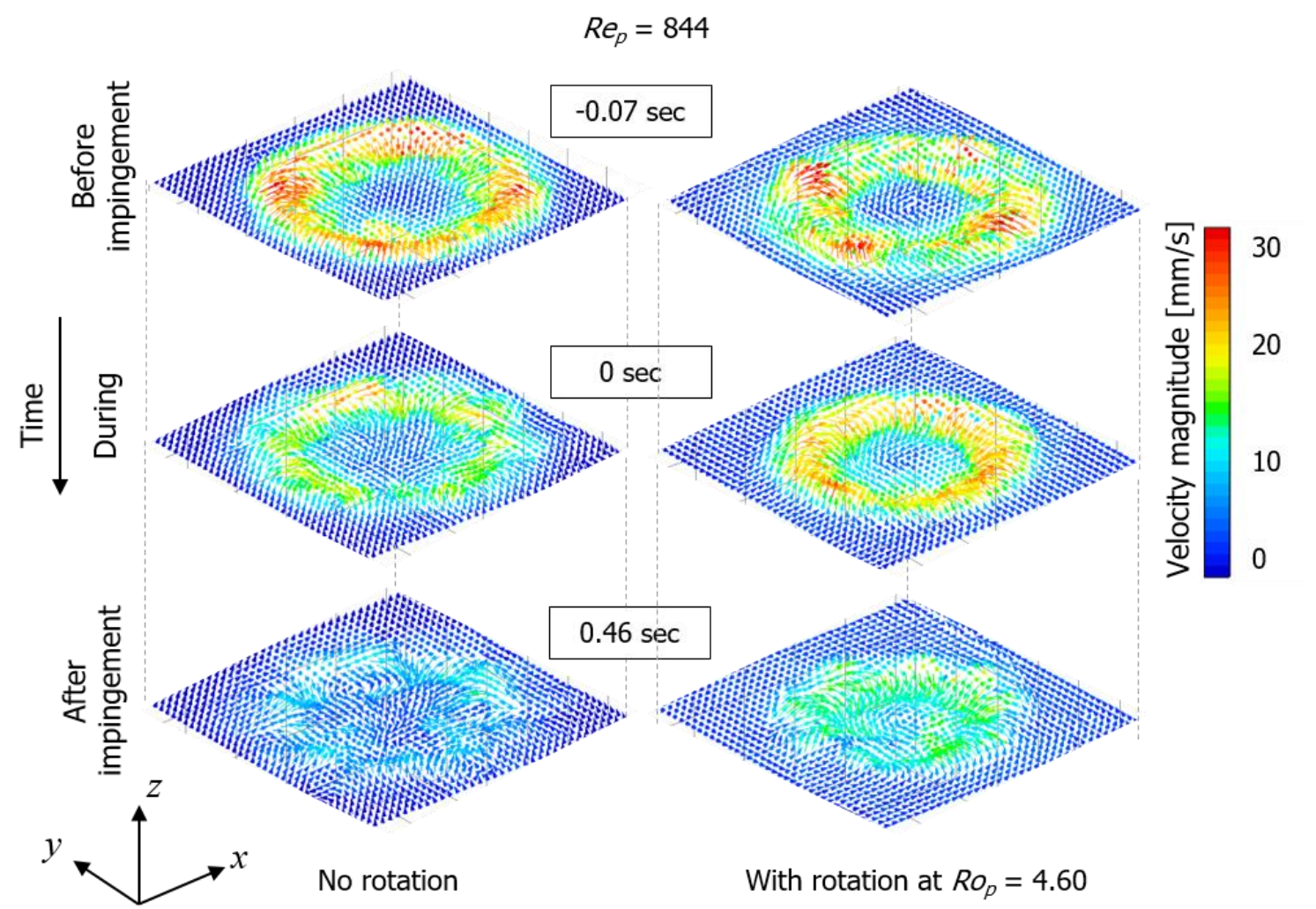

Fig. 3 Samples of velocity vector distributions obtained by PTV with interpolation applied for $\operatorname{Re}_{P}=844$

\subsection{Three-dimensional vortex structures}

Figure 4 shows vorticity distributions in $x-y$ plane, i.e. axial vorticity, $\omega_{z}$, in the process of wall impingement from the velocity vector data shown in Fig. 3. Here $\omega_{z}$ is defined by the relative value to the angular velocity of the rotating container $\Omega$. Thus, absolute vorticity is given by $\omega_{\mathrm{z}}+\Omega$. Positive vorticity $\left(\omega_{\mathrm{z}}\right.$ $>0)$ colored in red in the figure indicates dominance of cyclonic swirl while negative region in blue corresponds to anti-cyclonic structure. To prove the above interpretation at the condition of $\operatorname{Re}_{P}=844$ and $R o_{P}=4.60$, all the three components of vorticity is analyzed on every time frame. Fig. 4 shows isosurfaces of cyclonic and anti-cyclonic structure measured from the axial vorticity $\omega_{z}$. The wall impingement occurs at $t=$ 0 . Here the timing $t=0$ is estimated from the distance of vortex ring core from the wall, divided by the translational speed of vortex ring in approaching stage (This is a superficial time of the wall impingement since vortex core does not actually contact with the wall). Without background rotation shown in Fig. 4(a), many threads of vorticity take place both in positive and negative values after the impingement. These fine structures promote turbulent diffusion so that vortex ring is dissipated soon. Applying background rotation as shown in Fig. 4(b), it starts with cyclonic swirl on its head, and then wears an anti-cyclonic layer. As further time elapses, a new anti-cyclonic core is generated and left at the central axis. 


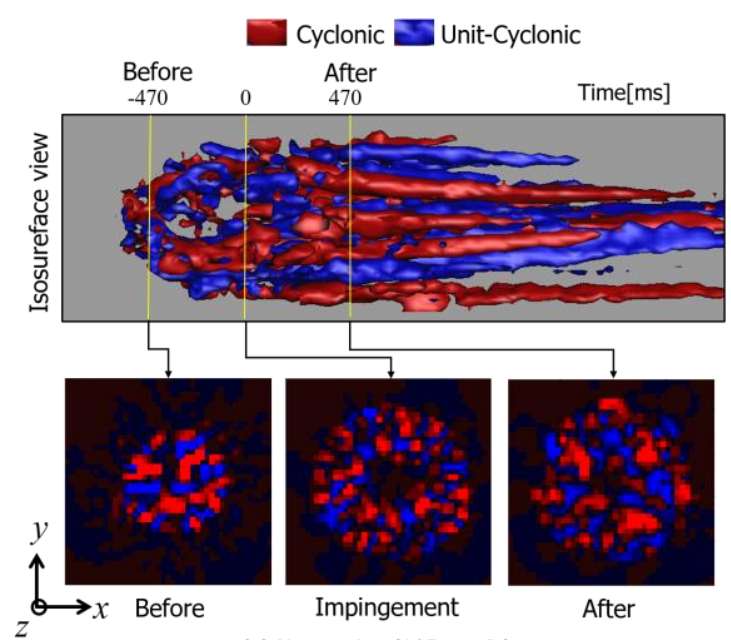

(a) No rotation $\left(1 / R o_{p}=0\right)$

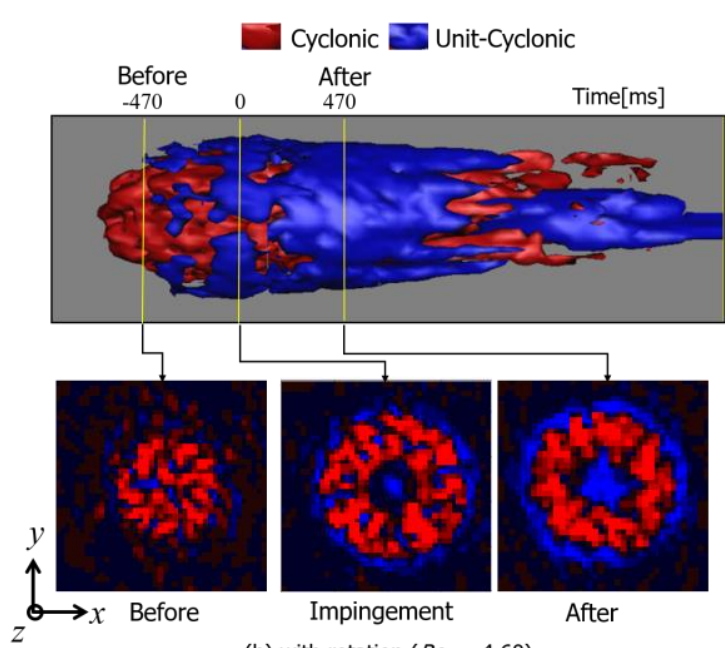

(b) with rotation $\left(R o_{P}=4.60\right)$

Fig. 4 Space-time 3D distribution of axial vorticity $\omega_{z}$ for vortex ring at $R e_{p}=844$. Red regions have positive vorticity which corresponds with cyclonic swirl in the case with background rotation. (a) No rotation, (b) with rotation at $R o_{p}=4.60$.

Figure 5 shows space-time 3D structure of azimuthal vorticity, $\omega_{\theta}$. This vorticity component represents the primary intensity of vortex tube that orients in the azimuthal direction. Here we obtain this vorticity component from PTV data on $x-y$ plane, $u(x, y, t)$ and $v(x, y, t)$, using the following relations,

$$
\omega_{\theta}=-\omega_{x} \sin \theta+\omega_{y} \cos \theta,\left\{\begin{array}{l}
\cos \theta=\left(x-x_{0}\right) / \sqrt{\left(x-x_{0}\right)^{2}+\left(x-x_{0}\right)^{2}} \\
\sin \theta=\left(y-y_{0}\right) / \sqrt{\left(x-x_{0}\right)^{2}+\left(x-x_{0}\right)^{2}}
\end{array}\right.
$$

where two vorticity on the cartesian coordinate are defined by

$$
\omega_{x}=\frac{\partial v}{\partial z}-\frac{\partial w}{\partial y}, \quad \omega_{y}=-\frac{\partial u}{\partial z}+\frac{\partial w}{\partial x}
$$

to which, further, continuity equation is applied to obtain the out-of-plane velocity, $w$, i.e.

$$
\frac{\partial u}{\partial x}+\frac{\partial v}{\partial y}+\frac{\partial w}{\partial z}=0, \quad \rightarrow \quad w(x, y, t)=\int_{0}^{h}-\left(\frac{\partial u}{\partial x}+\frac{\partial v}{\partial y}\right) d z
$$

The above integral equation for $w$ is numerically obtainable using the boundary condition, $w=0$ on the wall. Accuracy of the out-of-plane velocity, i.e. wall-perpendicular velocity depends on two factors: PTV quality against spatial differentiation, and the distance of the PTV plane from the wall, $h=10 \mathrm{~mm}$. The former is guaranteed by the higher order spatial interpolation of velocity vector field with $C_{3}$ continuity as explained previously. For the later factor, we need to reduce $h$ so that the shortest length of the flow structure is resolved. A similar technique was reported by our group for a planar PIV applied for 3D thermal convection in a thin layer (Takahashi et al. 2010). For the error level of $w$, we estimate it to be $20 \%$ in maximum case, judged from a preliminary experiment. Since $w$ is generally much slower than $u$ and $v$ due to wall proximity, the error of $w$ relative to the representative in-plane velocity is further regulated, and can be estimated to be $5 \%$ in the 
present measurement configuration.

As seen at the bottom three panels in Fig. 5, the azimuthal vorticity well visualizes the main body of the vortex ring in red color. In the case without background rotation, the main body of the ring accompanies an azimuthal wave as it approaches the wall (Fig. 5(a)). With background rotation, in contrast, the azimuthal wave is erased from the main body after the impingement (Fig. 5(b)). At the same time, negative azimuthal vorticity shown by blue layer is left around the main body. This infers persistence of axisymmetric two-dimensional structure of the vortex ring due to the background rotation. It also leads to expansion of the life time of the main body (of which perspective was reported by Ponitz et al. (2016) as being evidenced by elongation of the red-colored isosurface in the upper contour figures.

Figure 6 shows the radial vorticity component, $\omega_{r}$, representing appearance of longitudinal vortices at wall proximity in the radial direction. In the case without background rotation (see Fig. 6(a)), the radial vorticity gradually organizes a stripe pattern in the azimuthal direction during the wall impingement. The pattern has a similarity to the one observed in the axial vorticity distribution. This evidences the formation of a countable number of the longitudinal vortices on the wall. As visualized in the isosurface figure, the longitudinal vortices remain long in time after the impingement. To the contrary, such a clear structure disappears as applying background rotation (see Fig. 6(a)). It is generally known that longitudinal vortices enhance wall shear stress and heat transfer on the wall. The disappearance of them in the background rotation implies that the energy dissipation during wall impingement is suppressed by the rotation effect.
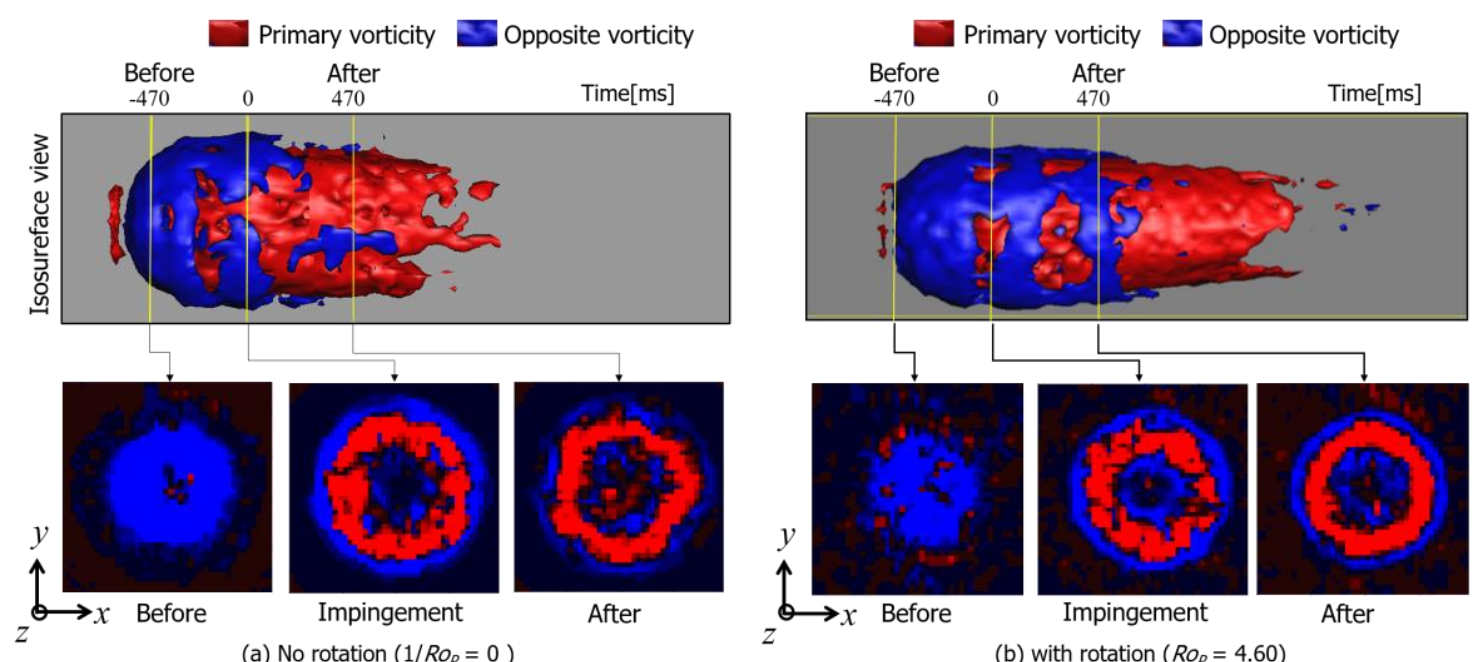

Fig. 5 Space-time 3D distribution of azimuthal vorticity $\omega_{\theta}$ for vortex ring at $\operatorname{Re} P=844$. Red regions have positive vorticity corresponding with primary vorticity of the vortex ring core. (a) No rotation, (b) with rotation at $R_{0 P}=4.60$. 


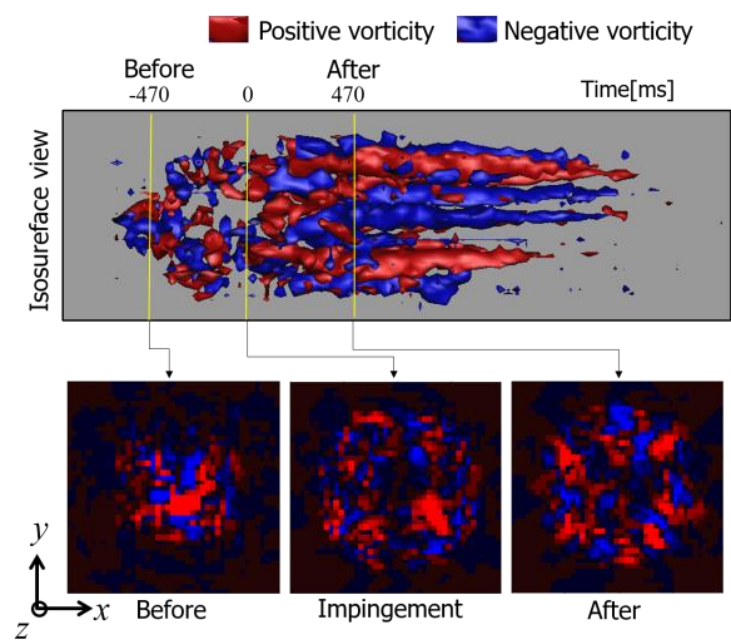

(a) No rotation $\left(1 / R o_{p}=0\right)$

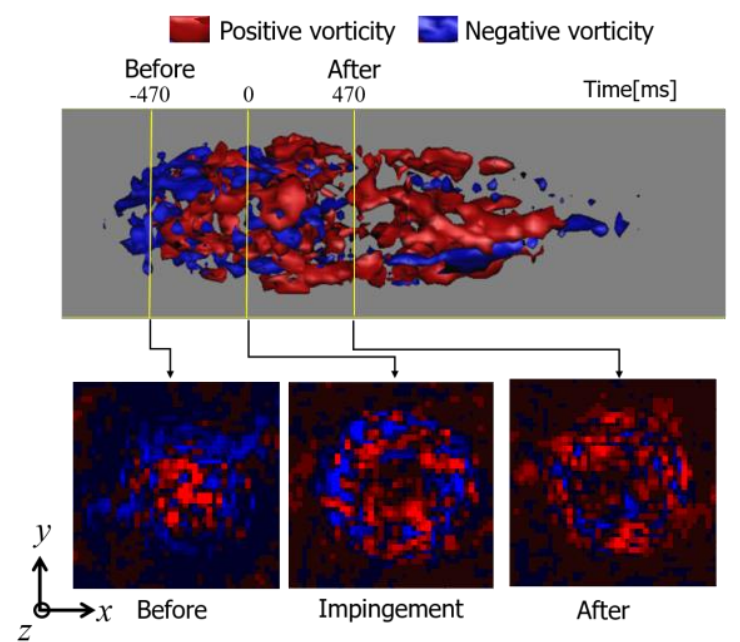

(b) with rotation $\left(R o_{p}=4.60\right)$

Fig. 6 Space-time 3D distribution of radial vorticity $\omega_{r}$ for vortex ring at $R e_{P}=844$. Positive vorticity shown in red corresponds with vortical motion o fluid between the wall and the axial cyclonic swirl induced above the primary vortex ring cores. (a) No rotation, (b) with rotation at $R o_{P}=4.60$.

\subsection{Interpretation of 3D structure}

Summarizing above-mentioned items visualized by three vorticity components, we are confident of concluding that the background rotation provides anti-effect to 3D development, and this nature resists against turbulent collapse of vortex ring in wall-impingement process. Our final interpretation read from the present results has been figured out graphically into Fig. 7. Although experimental information is limited and still incomprehensive, following interpretation could be described. In free-travelling process, a vortex ring has a primary mode with the azimuthal vorticity $\omega_{\theta}$. It accompanies Widnall instability waves, inducing a fluctuation of the radial vorticity $\omega_{r}$. When the vortex ring approaches to the bottom wall, the fluctuation of $\omega_{r}$ is intensified to further induce the axial vorticity $\omega_{z}$. Soon after then, it transits to a turbulent state in which all three vorticity components interact mutually. To the contrary, this mechanism is replaced with new one when the whole system is subject to background rotation, $\Omega$. Firstly, $\Omega$ induces cyclonic column as fluid migrates inwards during wall impingement. The column has large value in $\omega_{\mathrm{z}}$ in the central part, due to conservation of circulation, azimuthal fingering come from Widnall instability is overshadowed by the anti-cyclonic layers formed around the column. This stops transition to three-dimensional collapse, and rather stabilizes the primary structure of $\omega_{\theta}$.

Fig. 7 Schematic notation of multi-polar vortex dynamics for the wall-impinging vortex ring with background rotation, having been understood from the present experimental investigations.

Fig. 7 Schematic notation of multi-polar vortex dynamics for the wall-impinging vortex ring with background rotation, having been understood from the present experimental investigations. 


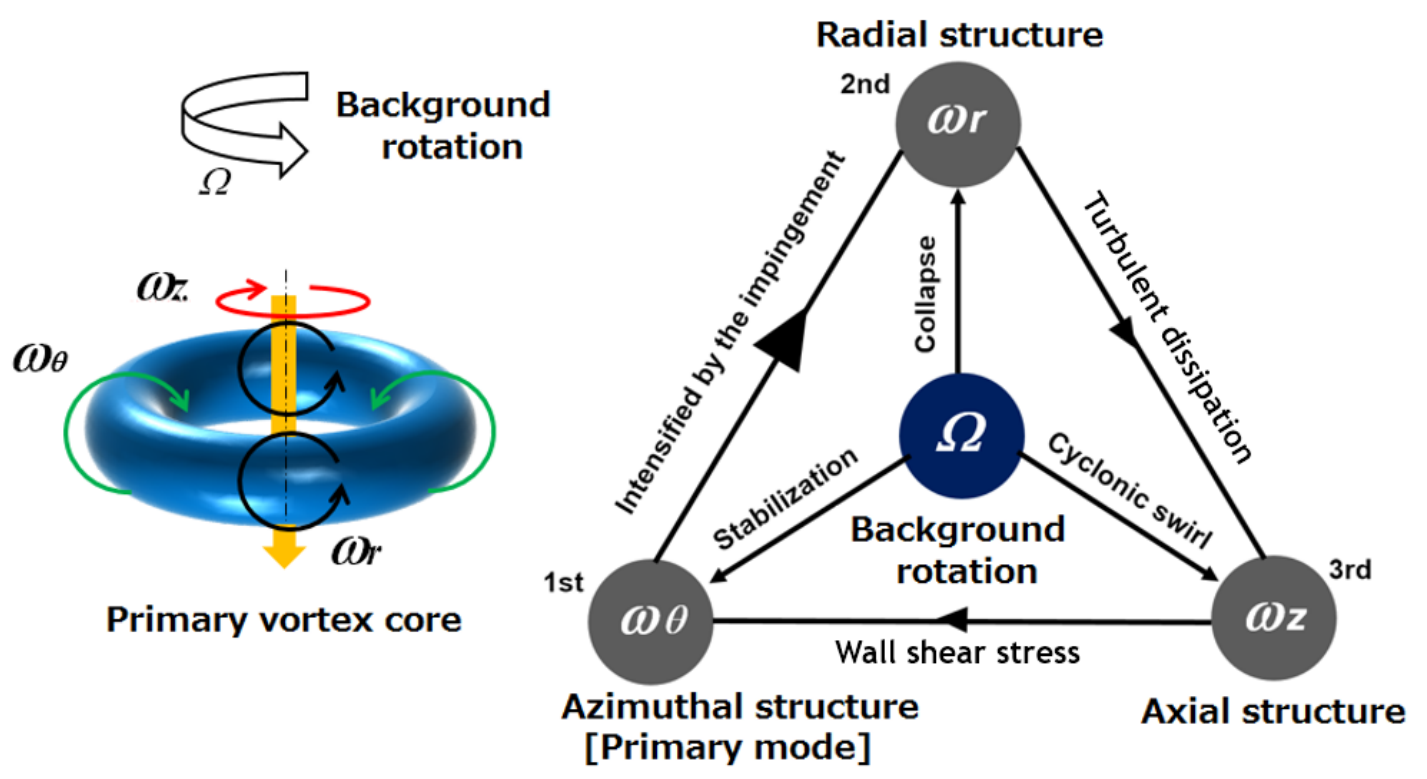

Fig. 7 Schematic notation of multi-polar vortex dynamics for the wall-impinging vortex ring with background rotation, having been understood from the present experimental investigations.

\section{Concluding Remarks}

In this paper, we reported how vorticial structure of a single vortex ring transits during flat wall impingement as the system is subject to background rotation. The investigation was based on flow visualization experiments coupled with particle tracking velocimetry (PTV). Regarding the parameter space, we focused on a competition regime in which translational inertia of the vortex ring and centrifugal force due to the background rotation interacted with each other. One of the experimental findings from the visualization is that the azimuthal waves of vortex ring are reduced as the background rotation is applied. Instead, a cyclonic column was formed during the wall impingement. To explain these trends quantitatively, we measured all three components of vorticity, $\omega_{r}, \omega_{z}$, and $\omega_{\theta}$ from the PTV data as functions of both time and space. From the results, we could obtain individual interaction patterns among the three components as discussed in the previous chapter. From the vorticity isosurfaces, we found that the main body of the vortex ring survives on wall impingement and stays in axisymmetric structure longer than the case without background rotation.

\section{Acknowledgments}

We acknowledge to Mr. Yuichi Nambu and Mr. Yuki Aikawa for their technical supports in measurement instrumentation.

\section{References}

Arévalo G, Hernández RH, Nicot C, Plaza F, (2010) Particle image velocimetry measurements of vortex rings head-on collision 
with a heated vertical plate. Phys Fluids 22: 053604.

Auerbach D (1988) Some open questions on the flow of circular vortex rings. Fluid Dyn Res 3: 209-213.

Bethke N, Dalziel SB (2012) Resuspension onset and crater erosion by a vortex ring interacting with a particle layer. Phys Fluids 24: 063301.

Boldes U, Ferreri JC (1973) Behavior of vortex rings in the vicinity of a wall. Phys Fluids, 16(11): 2005-2006.

Brend MA, Thomas PJ, (2009) Decay of vortex rings in a rotating fluid. Phys Fluids 21(4): 44105.

Cheng M, Lou J, Lim TT (2014) A numerical study of a vortex ring impacting a permeable wall. Phys Fluids 26:103602.

Chu CC, Wang CT, Chang CC (1995) A vortex ring impinging on a solid plane surface-Vortex structure and surface force. Phys Fluids 7(6):1391-1401.

Dazin A, Dupont P, Stanislas M (2006) Experimental characterization of the instability of the vortex ring. Part I:linear phase. Exp Fluids 40:383-399.

Dziedzic M, Leutheusser HJ (1996) An experimental study of viscous vortex rings. Exp Fluids 21:315-324

Gargan-Shingles C, Rudman M, Ryan K, (2015) The evolution of swirling axisymmetric vortex ring, Phys Fluids 27: 087101.

Gharib M, Rambod E, Shariff K, (1998) A universal time scale for vortex ring formation. J Fluid Mech 360:121-140

Glezer A (1988) The formation of vortex rings. Phys Fluids 31:3532-3542

Ido T, Murai Y (2006) A recursive interpolation algorithm for particle tracking velocimetry, Flow Meas Instr, 17: 267-275.

Ido T, Murai Y, Yamamoto F (2002) Post-processing algorithm for particle tracking velocimetry based on ellipsoidal equations, Exp Fluids 32: 326-336.

Ishikawa M, Murai Y, Wada A, Iguchi K, Yamamoto F (2000) A novel algorithm for particle tracking velocimetry using the velocity gradient tensor. Exp Fluids 29 (6): 519-531.

Jambunathan K, Lai E, Moss MA, Button BL (1992) A review of heat-transfer data for single circular jet impingement. Int J Heat Fluid Flow 13: 106-115.

Kitaura H, Murai Y, Takeda Y, (2010) Thomas PJ, Velocity vector field measurement of vortex rings using UVP. Trans Japanese Society of Mech Eng, 76:2143-2151.

Krueger PS, Gharib M (2003) The significance of vortex ring formation to the impulse and thrust of a starting jet. Phys Fluids $15: 1271-1281$

Krueger PS (2005) An over-pressure correction to the slug model for vortex ring circulation. J Fluid Mech 545:427-443.

Linden PF, Turner JS (2001) The formation of 'optimal' vortex rings and the efficient of propulsion devices. J Fluid Mech $427: 61-72$

Luton JA, Ragab SA (1997) The three-dimensional interaction of a vortex pair with a wall. Phys Fluids 9(10): 2967-2980

Naaktgeboren C, Krueger PS, Lage JL (2012) Interaction of a laminar vortex ring with a thin permeable screen. J Fluid Mech 707: $260-286$ 
Naitoh T, Okura N, Gotoh T, Kato Y (2014) On the evolution of vortex rings with swirl. Phys Fluids 26: 067101

Naguib AM, Koochesfahani MM (2004) On wall-pressure sources associated with the unsteady separation in a vortex-ring/wall interaction. Phys Fluids, 16(7), 2613-2622

Norbury J (1973) A family of steady vortex ring. J Fluid Mech 57:417-431

Martin H (1977) Heat and mass transfer between impinging gas jets and solid surfaces. Adv Heat Transf. 13: 1-60

Masuda N, Yoshida J, Ito B, Furuya T, Sano O (2012) Collision of a vortex ring on granular material, part I: interaction of the vortex ring with the granular layer. Fluid Dyn Res 44: 015501

Misaka T, Holzäpfel F, Hennemann I, Gerz T, Manhart M, Schwertfirm F (2012) Vortex bursting and tracer transport of a counter-rotating vortex pair. Phys Fluids 24: 025104

Moffatt HK (1988) Generalised vortex rings with and without swirl. Fluid Dyn Res 3: 22-30

Mohseni K, Gharib M (1998) A model for universal time scales of vortex ring formation. Phys Fluids 10:2436-2438

Mujal-Colilles A, Dalziel SB Bateman A (2015) Vortex rings impinging on permeable boundaries. Phys Fluids 27: 015106

Murai Y, Tasaka Y, Oishi Y, Takeda Y (2018) Modal switching of bubbly Taylor-Couette flow investigated by particle tracking velocimetry, Exp Fluids 59: 164

Murai Y, Vlaskamp JHA, Nambu Y, Yoshimoto T, Brend MA, Denissenko P, Thomas PJ, (2013) Off-axis PTV for 3-D visualization of rotating columnar flows. Exp Thermal Fluid Sci 51: 342-353

Orlandi P, Verzicco R (1993) Vortex rings impinging on walls: axisymmetric and three-dimensional simulations. J Fluid Mech 256:615-646

Ponitz B, Sastuba M, Brücker C (2016) 4D visualization study of a vortex ring life cycle using model analyses, J Vis, 19: 237-259.

Saffman PG (1970) The velocity of viscous vortex rings. Studies in Applied Math, XLIX, 4:371-380

Schram C, Riethmuller ML (2001) Vortex ring evolution in an impulsively started jet using digital particle image velocimetry and continuous wavelet analysis. Meas Sci Tech 12: 1413-1421

Shariff K, Leonard A (1992) Vortex rings. Ann Rev Fluid Mech 24: 235-279

Suzuki A, Kumagai I, Nagata Y, Kurita K, Barnouin-Jha OS (2007), Modes of ejecta emplacement at Martian craters from laboratory experiments of an expanding vortex ring interacting with a particle layer, Geophys. Res. Lett., 34 (2007), p. L05203

Swearingen JD, Crouch JD, Handler RA (1995) Dynamics and stability of a vortex ring impacting a solid boundary. J Fluid Mech 297:1-28

Takahashi J, Tasaka Y, Murai Y, Takeda Y, Yanagisawa T (2010) Experimental study of cell transition induced by internal heat sources in a shallow fluid layer. Int J Heat Mass Transfer, 53: 1483-1490.

Tung C, Ting L, (1967) Motion and decay of a vortex ring. Phys Fluids 10:901-910

Turkington B (1989) Vortex rings with swirl: axisymmetric solutions of the Euler equations with nonzero helicity. SIAM J 
Mathematical Analysis, 20(1):57-73.

Verzicco R, Orlandi P, Eisenga AHM, van Heijst GJF, Carnevale GF (1996) Dynamics of a vortex ring in a rotating fluid. J Fluid Mech 317:215-239.

Walker JDA, Smith CR, Cerra AW, Doligalski TL (1987) The impact of a vortex ring on a wall. J. Fluid Mech 181:99-140.

Weigand A, Gharib M, (1994) On the decay of a turbulent vortex ring. Phys Fluids 6: 3806-3808

Widnall SE, Bliss DB, Tsai CY (1974) The instability of short waves on a vortex ring. J Fluid Mech 66:35-47

Xu Y, He GS, Kulkarni V, Wang JJ (2017) Experimental investigation of influence of Reynolds number on synthetic jet vortex ring impinging onto a solid wall. Exp Fluids 58: 6.

Yamada H, Kohsaka T, Yamabe H, Matsui T (1982) Flowfield produced by a vortex ring near a plane wall, J. Physical Society of Japan, 51(5):1663-1670. 